\title{
VIRTUAL FRACTURE CLINIC: A PANDEMIC-READY TOOL FOR IMPROVING THE EFFICIENCY OF FRACTURE CLINIC
}

\author{
Bonnie McRael*, Nicholas Shortt', Natalie Campbell', Christopher Burton', Justin Scott2, \\ Jane Fitzpatrick ${ }^{3}$ \\ 1. Logan Hospital, Metro South Health Services (Queensland Health), Loganholme QLD, Australia \\ 2. QCIF Facility for Advanced Bioinformatics, Institute for Molecular Bioscience, The University of Queensland, Australia \\ 3. Centre for Health and Exercise Sports Medicine, Faculty of Medicine, Dentistry and Health Science, University of Melbourne, \\ Australia
}

Correspondence: drbonniemcrae@gmail.com

\begin{abstract}
BACKGROUND:

The traditional model of care of the Orthopaedic Fracture Clinic (OFC) is labour intensive, expensive, has poor satisfaction rates, and often has minimal impact on management and outcomes of patients with minor injuries. Our aim was to implement a Virtual Fracture Clinic (VFC) for the management of minor injuries that is safe, reduces OFC clinic workload and reduces the OFC failure to attend (FTA) rate.
\end{abstract}

\section{METHODS:}

This study was a retrospective longitudinal audit of OFC workload before (January 2012 -February 2017) and after (March 2017 - December 2019) implementation of the VFC. It was performed in an urban district general hospital in South East Queensland, Australia. The primary outcome measures included attendances per timepoint (month).

\section{RESULTS:}

Overall, we observed a significant reduction in total number of patients from 1,055 (IQR 104.5) to 831 (IQR: 103) per month coming through the OFC following the introduction of the $\operatorname{VFC}(F=21.9 ; \mathrm{df}=1 ; p<0.0001)$. The failure to attend rate was reduced by $44 \%$ from 271 (IQR: 127.3) to 151 (IQR: 72.8) ( $F=4.0 ; d f=1 ; p=0.047)$.

\section{CONCLUSION:}

The VFC implementation was successful in improving efficiency and reducing the current OFC workload, as well as reducing FTA rate. Reduction in clinic workload allows more time to be spent with complex patients, prevents clinic backlogs and overbooking, and crowding of waiting rooms. In the midst of a global pandemic that is spread by close contact, virtual clinics seem the way of the future to treat patients whilst minimising risk of COVID-19 spread.

\section{KEYWORDS}

Virtual fracture clinic, acute trauma, fracture clinic, orthopaedic outpatient 


\section{INTRODUCTION}

In an era where hospitals are striving for healthcare-delivery efficiency and cost-effectiveness, many medical and surgical specialties are seeking ways to streamline the outpatient clinic experience both for patients and clinicians. This is particularly true in Orthopaedics, where the classic model of Orthopaedic Fracture Clinic is labourintensive for both clinical and administrative staff, and patients often experience extensive wait times due to overbooking. Overbooking stems from a combination of factors: increasing demand for services supplied by a limited capacity clinic, as well as clinic booking templates that often do not reflect the true clinic capacity (a product of clinician time and rooms available).[1] The common practice of 'overbooking' clinics is used to compensate for the proportion of patients who will fail to attend (FTA) their appointment. [1] It leads to more crowded waiting rooms and longer waiting times at the clinic. Overbooking in the OFC also stems from a large proportion of ED referrals, which creates a substantial burden on the clinic.

Currently, Queensland public hospitals undertake on average 105,624 orthopaedic outpatient appointments each year (the highest of all specialist outpatient services). Increasing population and life expectancy, coupled with an increasing burden of disease and disability, is resulting in increased demand for specialist orthopaedic services. Demand is expected to outweigh capacity and, in an ever increasing fiscally challenged environment, health services and clinicians need to ensure optimal use of resources to meet the needs of all Queenslanders. [2]

In 2016, our OFC saw 17,195 patients, of which 5,466 (31.8\%) were new referrals from ED. (3) In a regular clinic there are expected to be 50 patients per day. With the overload there are an additional 16 patients per day. The clinic is staffed by junior doctors consisting of residents, training and non-training registrars, with backup advice from a consultant in a parallel clinic. The patient load is 25 per four hours, and with the additional patients this becomes 33 per four-hour clinic. This results in clinics frequently running over time and reduced consultation time for each patient as staff attempt to keep running to schedule. A study looking at factors influencing patient satisfaction with orthopaedic outpatient clinic identified key environmental factors resulting in dissatisfaction as increase clinic wait time, and reduced or insufficient contact time with the clinician. [4] Thus, an alternative and innovative model of care for reduced face-to-face attendances, particularly during the COVID pandemic, is required to provide sustainable, patient focused and quality outcome driven services for the orthopaedic outpatient department.

The traditional patient population attending the OFC includes patients with acute fractures, but also includes all musculoskeletal injuries requiring outpatient follow up (Figure 1). Fracture-related appointments are expected to be scheduled within 1-2 weeks of the acute injury to ensure timely intervention in those patients with complications such as malpositioned or unstable fractures. Unfortunately, for many patients without these complex fracture issues, the review occurs in the acute period whilst the patient still has substantial pain and immobility, resulting in less than adequate assessment upon initial review. The patient is subsequently brought back for review once the acute symptoms have settled to allow for thorough assessment, and then repeatedly reviewed over the following weeks to observe fracture healing. Multiple review appointments for a single patient increases the demand on the capacity of the OFC. Increasing demand for appointments has been observed with simple, stable, self-limiting musculoskeletal injuries where initial management in the ED could be followed by referral to allied health professionals or the GP for management rather than increasing the workload of the OFC. $[3,5-8]$ 


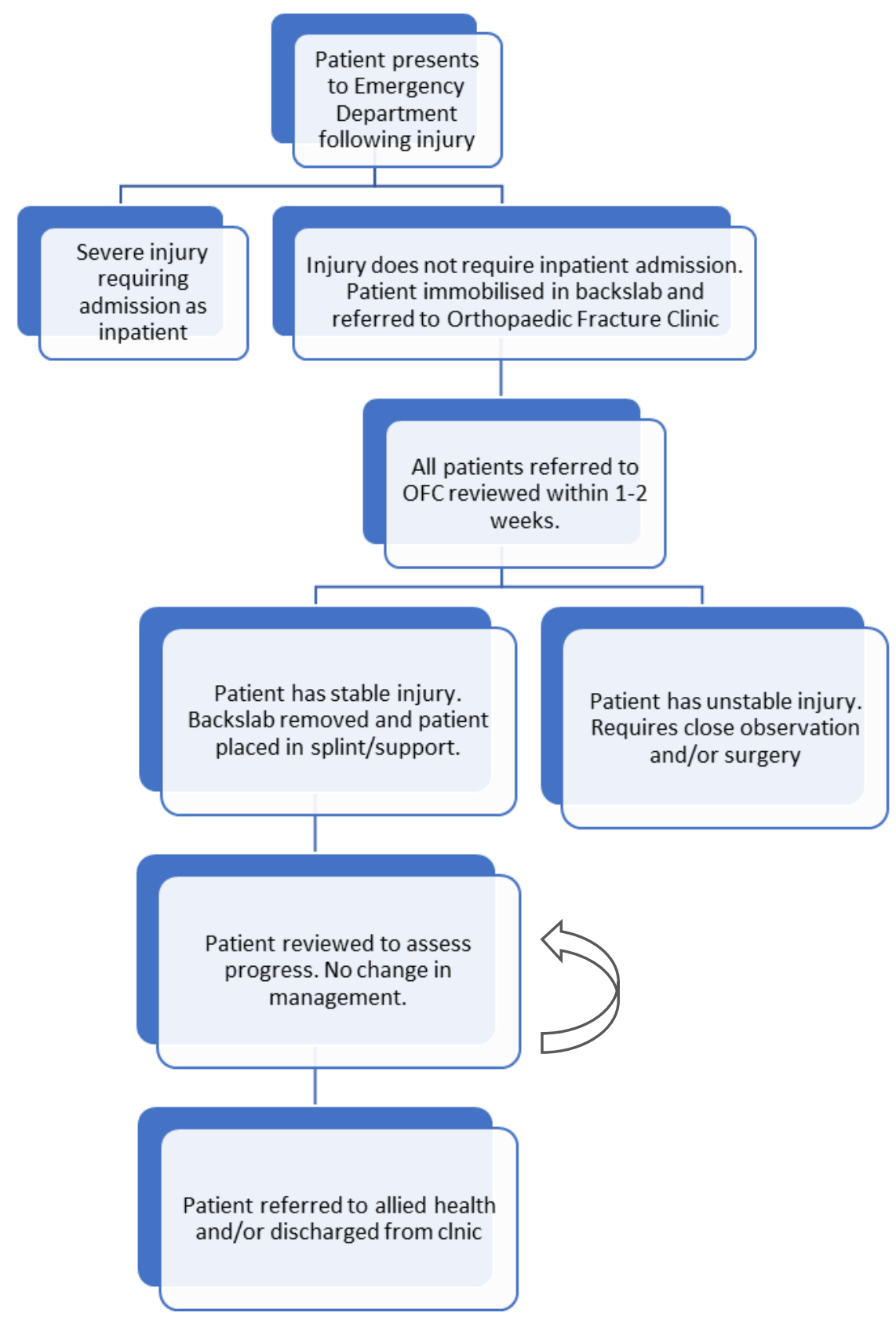

Early review of patients with acute fractures is also directed by the traditional model of care where the majority of fractures are immobilised in a Plaster of Paris (POP) backslab. Patients are asked to attend clinic during the acute injury period to assess the integrity and adequacy of the POP that has been applied, or to remove the POP in instances where it has been applied injudiciously to injuries that could be managed in other ways. Unfortunately, immobilisation can lead to stiffness and reduced function, which can be a cause for concern for patients and inexperienced junior clinicians, and subsequently results in patients being asked to return to OFC for review to reevaluate their movement after a period of time.
The creation of a Virtual Fracture Clinic Model of Care at the Glasgow Royal Infirmary [9] sought to solve the problems posed by the traditional OFC model and the concept is being adopted in Orthopaedic units internationally. [6-8, 10] In the Glasgow model, patients seen in the ED with fractures not requiring admission are given education on their specific injury and referred to VFC. At the VFC the case notes and radiographs are reviewed by an orthopaedic consultant and a telephone consultation with the patient follows to discuss the management strategy. There are three outcomes from the VFC: referral back to the GP or to a physiotherapist, a faceto-face review in the OFC, or a review in a sub-specialty orthopaedic clinic. A growing body of literature highlights the role advanced physiotherapists can play in supporting 
Orthopaedics, through either completion of the VFC review under supervision or management of patients (both faceto-face and virtually) diverted by the VFC doctor. [1 1, 12]

In an audit of Logan Hospital OFC in 2016 2,216 patients attended the clinic over a four-week period. Of these, 1,041 (47\%) had simple injuries that did not receive any additional intervention by the orthopaedic team beyond what was received in the $E D$ and were observed over a 6-8 week period. Logan Hospital sought to implement a virtual fracture Model of Care based on the outcomes at the Glasgow Royal Infirmary. The goal was to reduce OFC workload, reduce waiting times for OFC appointments, allow greater consultation time for review of patients with more complex injuries, and minimise outpatient clinic appointment costs to our healthcare facility. Additionally, it was anticipated that patients diverted from OFC to allied health would demonstrate clinically and statistically significant improvements in their QuickDASH scores and high satisfaction with the VFC process. The QuickDASH is a responsive and validated outcome measure in Orthopaedic upper limb trauma, however has not been evaluated specifically in relation to VFC conditions. [13]

\section{METHODS}

Study design: This study was a retrospective longitudinal audit of fracture clinic workload before and after implementation of the VFC. It was performed in an urban district general hospital in South East Queensland, Australia. Ethical approval was sought and granted by Metro South HREC. After discussion with a statistician, it was decided that best reflection of VFC influence would be demonstrated by comparing five-year data from the traditional clinic vs three-year data for VFC. By doing this we hoped to eliminate any outliers or seasonal variation and get a true reflection of the OFC workload prior to VFC implementation. Inclusion criteria were all patients referred to the Logan Hospital orthopaedic acute trauma outpatient service from January 2012 to December 2019 (a total of 96 months). The VFC was implemented in March 2017. Units of measurement included attendances per timepoint (month). Overall, 96 timepoints were analysed; data was missing for the first 62 timepoints for VFC.

Data Collection: Data was obtained via the hospital electronic computer systems (Appointment Scheduling
Information System and Elective Management System) and the outpatient appointment booking systems by the Metro South Outpatient Department Data Manager. Data was collated into a Microsoft Excel (2019) spreadsheet and the following variables were recorded: clinic referred to (OFC/VFC), patient appointment status (new patient/review patient), whether a patient failed to attend their appointment (FTA), and whether a patient was discharged following their OPD appointment. Data was also collected regarding the number of orthopaedic outpatients reviewed in clinic by the plaster technicians. Patients attending Advanced Practice Hand Therapy after VFC review in lieu of attending OFC had additional data related to initial and discharge QuickDASH scores and satisfaction scores recorded into a Microsoft Excel (2019) spreadsheet. The satisfaction survey consisted of four questions, relating to the patient's satisfaction with: waiting times, seeing advanced practice allied health in place of attending fracture clinic, the knowledge of the therapist providing treatment and the treatment provided. Patients could choose one of five responses ranging from very dissatisfied, dissatisfied, neutral, satisfied or very satisfied.

Primary outcome measures included the total number of patients seen in the OFC, the number of new and review patients seen in the OFC, the number of FTAs, and the number of patients discharged from OFC per month. Secondary outcome was the volume of work performed by the plaster technicians each month.

VFC described injuries were defined in partnership by the orthopaedic department and the ED and incorporated into the Care Pathway (Table 1). The care pathway included specific advice relating to plaster cast immobilisation; POP immobilisation was to be avoided and removable splints used instead. All patients were given a verbal explanation of their injury by the treating ED medical officer and an information leaflet relating to their injury, created by the orthopaedic department. Leaflets used simple language to describe the injury, the natural history of the injury and expected recovery timeframes. Patients were discharged home with a letter and leaflet for their GP. The ED medical officer completed an electronic intrahospital patient referral to VFC triggering a virtual appointment booking for the patient. 
TABLE 1: VFC APPROVED INJURIES AND MANAGEMENT PATHWAYS

\begin{tabular}{|c|c|c|}
\hline INJURY & MANAGEMENT & DISCHARGE DESTINATION \\
\hline $\begin{array}{l}\text { Paediatric buckle fracture of distal } \\
\text { radius and/or ulna (<12 years old) }\end{array}$ & Wrist splint & General Practitioner \\
\hline $\begin{array}{l}\text { Paediatric clavicle fracture }(<12 \\
\text { years old })\end{array}$ & Collar \& Cuff & General Practitioner \\
\hline $\begin{array}{l}\text { Acromioclavicular joint sprain } \\
\text { (Rookwood class } 1 \text { \& 2) }\end{array}$ & Collar \& Cuff & General Practitioner \\
\hline $\begin{array}{l}\text { Proximal humerus fracture in } \\
\text { elderly patient (>80) with severe } \\
\text { dementia }\end{array}$ & Collar \& Cuff & General Practitioner \\
\hline Ankle sprain & Ankle brace or moonboot & $\begin{array}{l}\text { Acute (<3 weeks) - } \\
\text { General Practitioner } \\
\text { Chronic (>3weeks) - } \\
\text { Physiotherapy }\end{array}$ \\
\hline Malleolar flake avulsion fracture & Ankle brace or moonboot & General Practitioner \\
\hline $\begin{array}{l}\text { 5th metatarsal base fracture or } \\
\text { other distal metatarsal fracture }\end{array}$ & $\begin{array}{l}\text { Hard-sole walking shoe or } \\
\text { moonboot }\end{array}$ & General Practitioner \\
\hline Toe fractures & Buddy strap + heel walker shoe & General Practitioner \\
\hline $5^{\text {th }}$ metacarpal fracture & Buddy strap + soft bandage wrap & Hand Therapy \\
\hline $\begin{array}{l}\text { Finger distal phalanx tuft fracture } \\
\text { or undisplaced phalangeal } \\
\text { fractures }\end{array}$ & $\begin{array}{l}\text { Protective splint (i.e. Zimmer splint } \\
\text { or Mallet splint) }\end{array}$ & Hand Therapy \\
\hline $\begin{array}{l}\text { Mallet finger (joint not subluxed } \\
\text { and }<30 \% \text { of articular surface } \\
\text { involved) }\end{array}$ & Mallet splint & Hand Therapy \\
\hline Finger volar plate avulsion injury & Buddy strapping & Hand Therapy \\
\hline $\begin{array}{l}\text { Undisplaced radial head/neck } \\
\text { fracture (Mason } 1 \text { or 2) }\end{array}$ & Broad arm sling & Hand Therapy \\
\hline
\end{tabular}

VFC is held five-days per week and is conducted by one orthopaedic registrar and overseen by a single consultant orthopaedic surgeon. Digital radiographs and patient case notes are reviewed, and an appropriate management plan is determined based on the nature of the injury. Management pathways include:

1. Discharge to care of GP
2. Discharge to an appropriate Allied Health department (Advanced Practice Hand Clinic (APHC) or Physiotherapy), or

3. Referral to OFC for in-person assessment (Figure 2). APHC is led by an Advanced Physiotherapist with experience and appropriate credentialling to independently manage patients with Orthopaedic conditions. Use of this skillset and framework to its full potential affords the VFC process the ability to divert a 
larger subset of trauma within the recognised pathway conditions. An Orthopaedic registrar is available via an oncall phone for real time second opinions on imaging, and there are four appointment spaces per week in a consultant Orthopaedic surgeon clinic reserved for hand therapy to escalate cases of concern.
Referrals deemed not appropriate for VFC (i.e. injury not defined by VFC pathway, incorrectly diagnosed injury, or immobilisation with POP requiring plaster technician or medical offer for removal) are referred to OFC. A letter is sent to both the patient and the GP to advise them of the outcome of their VFC appointment.

\section{FIGURE 2: LOGAN HOSPITAL VIRTUAL FRACTURE CLINIC MODEL OF CARE}

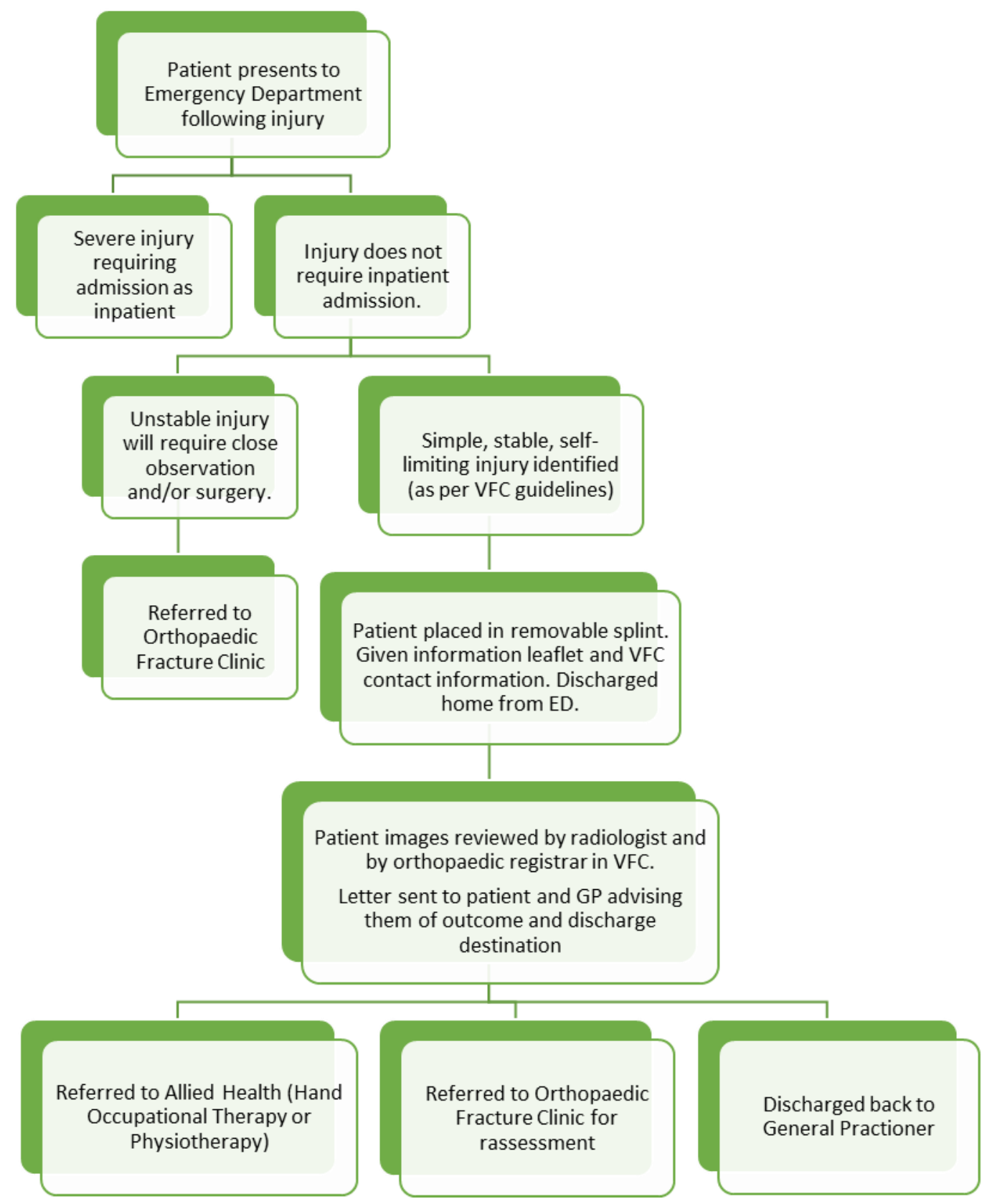

\section{STATISTICAL METHODS}

This analysis took the form of a retrospective interrupted time series (ITS). ITS is a useful tool for evaluating the effectiveness of healthcare interventions. Data is collected at time-points both before and after an intervention, to determine whether observed changes in outcome are explained by secular trends in the data, or are likely attributable to the intervention itself. [14] ITS compares the means before and after in a robust way, that takes into account correlations between time points and allows for 
controlling of confounders such as seasonality using a ANCOVA Lagged Dependent Variable model. [15]

The analyses were performed using the $R$ software version 4.0.2 (2020-06-22) and developed in RStudio 1.4.1103. [16, 17] The $R$ packages used were 'its.analysis' written by Patrick English and 'segmented' written by Vito M.R. Muggeo of which the 'its.analysis' package results are reported here for brevity. [18-20] For the variables of interest that were normally distributed for both clinic regimens, the mean and standard deviation was reported, otherwise the median and inter-quartile range was reported. The reported standard deviation for the OFC plaster technician appointments is an estimate only after correction for a missing value. This was an exploratory study, and no adjustments were made to the reported $p$-values to take into account multiple hypothesis testing.

\section{RESULTS}

A total of 96,741 patients were reviewed in the outpatient fracture clinics over the study period; 91,108 in OFC and 5,633 in VFC. The mean number of monthly OFC patients seen before and after implementation of the virtual clinic is shown (Figure 3). Overall, we observed a significant reduction in total number of patients (from 1,055 (IQR 104.5) to 831 (IQR: 103) per month) coming through the OFC following the introduction of the VFC $(F=21.9 ; \mathrm{df}=1 ; \mathrm{p}$ $<0.0001)$.

FIGURE 3: TIME SERIES CURVES OF TOTAL OFC PATIENTS SEEN PRE- AND POST- VFC IMPLEMENTATION

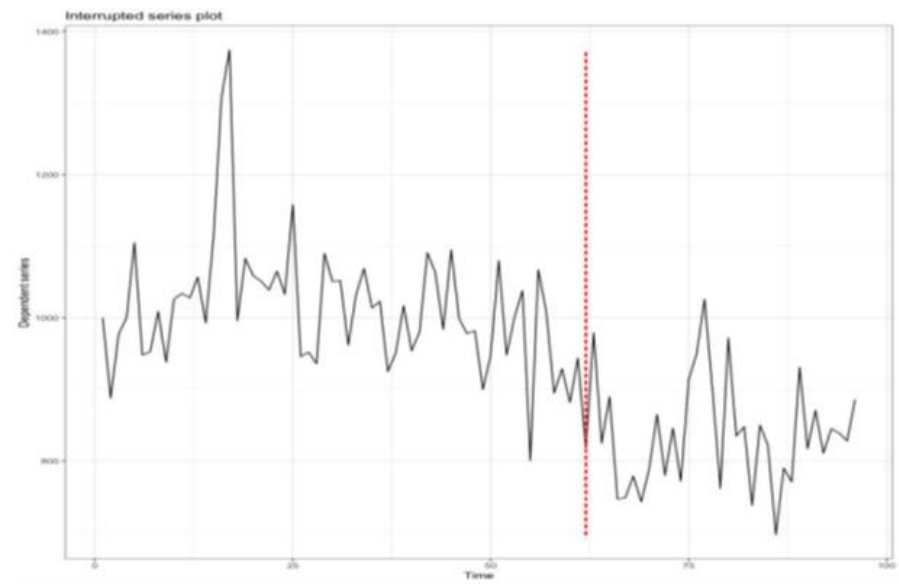

There was an $11 \%$ reduction in new patient referrals to OFC (407 (SD: 57.7) to 361 (SD: 48.4) (F=6.3; $d f=1 ; p=$ $0.014)$ ) and $20 \%$ reduction in review patients returning to
OFC (610 (SD: 96.6) to 484 (SD: 57.1) (F= 4.8; $d f=1 ; p=$ $0.032)$ ) (Figures 4 and 5).

FIGURES 4 AND 5: TIME SERIES CURVES OF NEW PATIENTS SEEN IN OFC AND REVIEW PATIENTS SEEN IN OFC
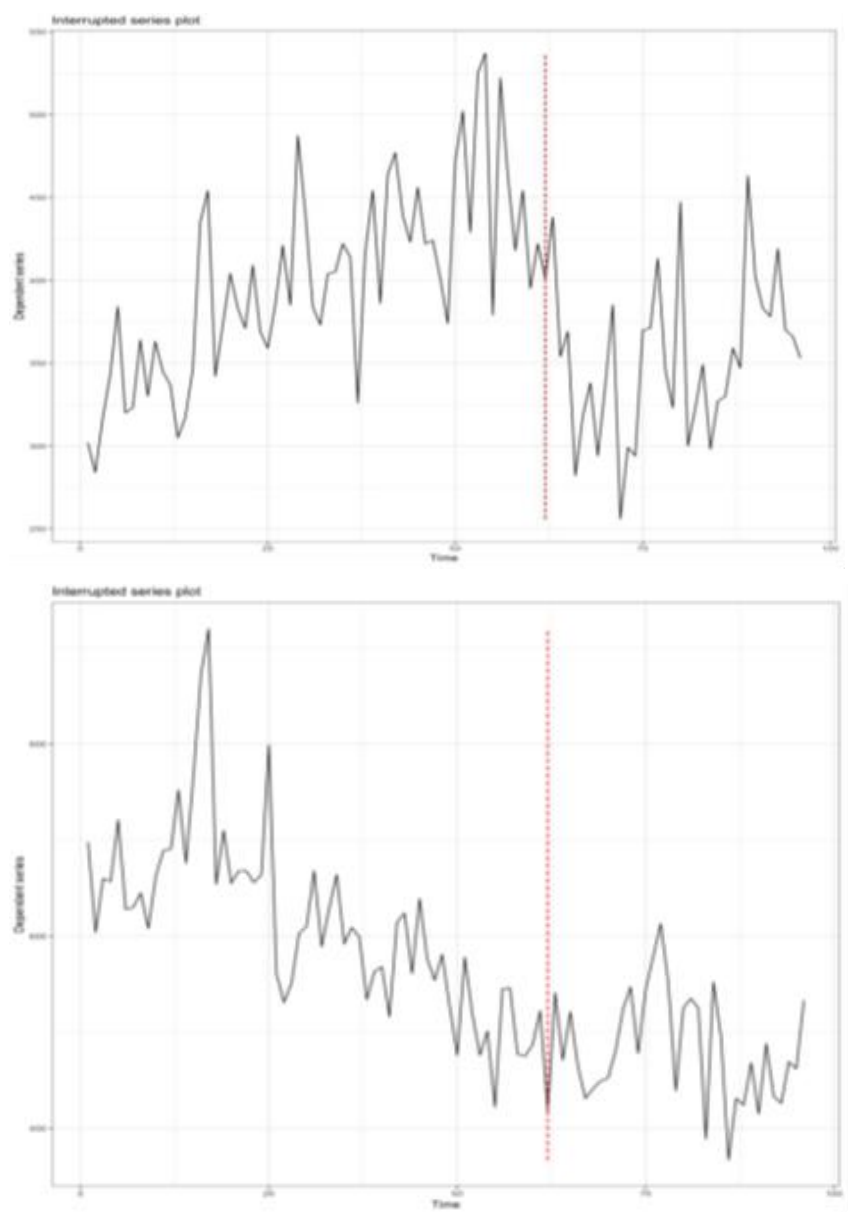

The FTA rate was reduced by $44 \%$ from 271 (IQR: 127.3) to 151 (IQR: 72.8) ( $F=4.0 ; d f=1 ; p=0.047)$ (Figure 6). A subanalysis was performed to determine the reduction in FTA rate in new OFC referrals versus review patients. There was no significant change in new patient failure to attend (from 113 (SD: 45.3) to 82 (SD: 33.3) ( $F=2.1 ; d f=1 ; p=0.151)$, in comparison to the larger significant reduction in OFC review patient FTA rates (from 169 (IQR: 49.2) to 86 (34) $(F=7.2 ; d f=1 ; p=0.009)$. This result correlates with our expectations about the VFC implementation; that the new patients attending OFC would have more severe injuries requiring orthopaedic consultation and are thus less likely to FTA. We hypothesised that the patients likely to FTA OFC pre-VRC implementation had either simple injuries not requiring intervention (new patients) or were reviewed at least once and then FTA as their condition improved (review patients). Implementation of the VFC will redirect both these patient populations to the virtual clinic. 
FIGURE 6: TIME SERIES CURES OF FTA IN ORTHOPAEDIC FRACTURE CLINIC

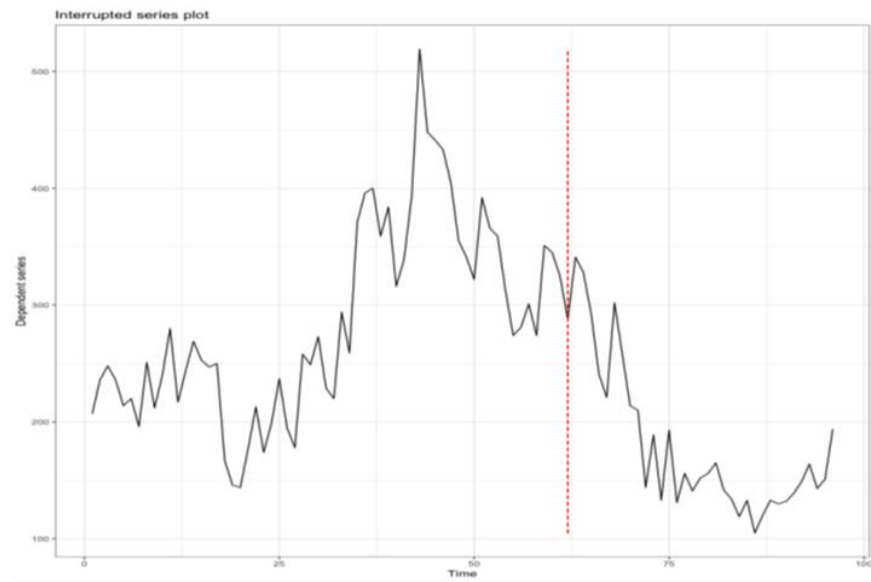

No significant change was noted in discharge rates from the OFC from 477 (IQR: 390) to 272 (IQR: 69.3) ( $p=0.124$ ) (Figure 7). A trend towards a significant change in median new patient discharges (176 (IQR: 168.5) to 91 (IQR: 39.8) (P $=0.076)$ ) was observed, and no significant changes in mean for review patients discharged from OFC (282 (IQR: 190) to 181 (IQR: 40.8) ( $p=0.236)$. This result is as expected, as the VFC was hypothesised to reduce the number of inappropriate OFC patient referrals; thus, the patients seen in OFC after VFC-implementation appropriately require face-to-face consultation with orthopaedic staff, and are not anticipated to be discharged at a significant rate from OFC.
FIGURE 7: TIME SERIES CURVES OF TOTAL NUMBER OF PATIENTS DISCHARGED FROM OFC

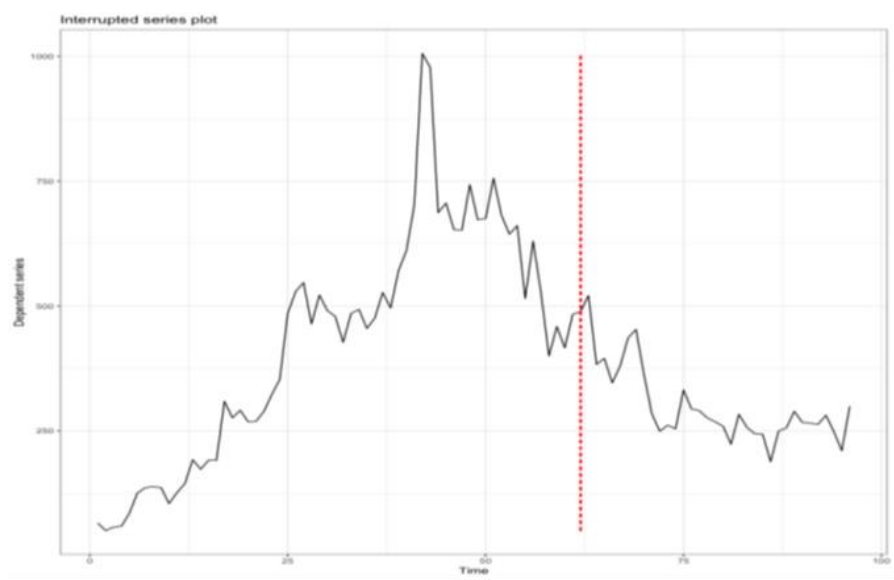

There was a significant change in plaster technician appointments after the VFC introduction from 482 (SD: 50) to 374 (SD: 50.4) ( $p<0.0001)$.

Of the patients referred to APHC following VFC review, 206 patients (July 2017 - February 2019) had complete data for the QuickDASH outcome. Average initial QuickDASH score was $64.3 \%$ whilst average discharge score was $11.3 \%$ resulting in a QuickDASH score improvement of $53 \%$. Satisfaction scores were collated between May 2017 to July 2019 with three hundred and thirty-six the lowest, and three hundred and fifty-two the highest number of responses to questions 1 to 4. Percentage of respondents indicating satisfied or very satisfied was ninety six percent to question 1, and ninety nine percent to question 2, 3 and 4 (Table 2).

\section{TABLE 2: APHC SATISFACTION SCORES}

\begin{tabular}{|l|l|l|l|l|l|}
\hline Patient Satisfaction Survey & $\begin{array}{l}\text { Very } \\
\text { Dissatisfied }\end{array}$ & Dissatisfied & Neither & Satisfied & $\begin{array}{l}\text { Very } \\
\text { Satisfied }\end{array}$ \\
\hline $\begin{array}{l}\text { 1. How satisfied were you with the waiting } \\
\text { time to receive your initial appointment in } \\
\text { the Advanced Practice Hand Clinic? }\end{array}$ & 1 & 6 & 5 & 82 & 258 \\
\hline $\begin{array}{l}\text { 2. How satisfied were you to see an } \\
\text { Advanced Practice Therapist rather than } \\
\text { a doctor at your initial appointment? }\end{array}$ & 1 & 1 & 2 & 70 & 264 \\
\hline $\begin{array}{l}\text { 3. How satisfied were you with the level of } \\
\text { knowledge of the Advanced Hand } \\
\text { Therapist who did your initial assessment? }\end{array}$ & 2 & 1 & 1 & 41 & 296 \\
\hline $\begin{array}{l}\text { 4. How satisfied are you with the } \\
\text { treatment you received? }\end{array}$ & 2 & $\mathbf{8}$ & $\mathbf{2}$ & 33 & 298 \\
\hline Total & & & & & \\
\hline
\end{tabular}




\section{DISCUSSION}

Value-based health care focuses on how to ensure sustainability of the system to meet the challenges of an aging population with increasing demand on the system, whilst recognising that a focus on efficiency alone is insufficient. The change in emphasis from volume to value challenges the system to better understand the patient experience, critically review how and where care is delivered, and reduce unwarranted clinical variation. [21] This virtual Model of Care has demonstrated its benefit during a global pandemic by reducing face-to-face consultations, as well as supporting the quadruple aim of value-based care through providing patients improved access to health services, better health outcomes, a satisfactory experience of their healthcare, as well as ensuring the health professionals involved are experiencing satisfaction in delivering such care and health system resources are better utilised. [22]

Jenkins et al. [9] described the potential advantages of a VFC as multifactorial; with benefits in the ED, Orthopaedic Departments and Allied Health, the potential to improve patient satisfaction, and potential healthcare cost savings. Although several countries have reported developing VFCs, the Logan Hospital VFC was one of the first virtual clinics to be implemented in Australia and has the largest cohort of data from our region. This study demonstrates the effectiveness of the clinic model of care in the Australian public health care system.

Implementation of a VFC model has shown value by reducing the number of face-to-face consultations in the OFC by $21 \%$, as well as allowing more efficient use of medical staff resources. [23, 24] This allows a single staff member to assess the VFC referrals, in significantly less time than would be required to review all the patients face-toface. This also reduces the time-pressure on doctors in the OFC, allowing for additional time to be spent appropriately with patients presenting with complex injuries requiring further work-up and management. These benefits have already been demonstrated at other sites by Legg et al. [6] McKirdy \& Imbuldeniya [25], Murray et al. [26] and O'Reilly et al. [8] Logan Hospital recorded an $11 \%$ reduction in new OFC referrals, in addition to a $20 \%$ reduction in the number of review appointments and a $44 \%$ reduction in FTAs. This has resulted in a decrease in the administrative burden following up such patients and consequently less overbooking of the OFC. This markedly improves overall efficiency and reduces waiting times for OFC patients. Additionally, and fortuitously, our audit has new relevance in that VFC can also assist with decreasing the in-person footprint in hospital clinics during pandemics such as COVID-19.

A number of previous studies have investigated the implementation of the VFC model with promising results relating to safety and efficiency. Holgate and colleagues [27] showed the VFC to significantly reduce the wait time for a face-to-face consultation within the OFC as well as being compliant with British Orthopaedic Association Standards for Trauma (BOAST 7 guidelines), thus demonstrating the safety of VFC. Cavka et al. [11] recorded a significant improvement in patient wait times for first contact with the orthopaedic team with the introduction of VFC, from a median of seven days to two. They also reported a reduced rate of unplanned ED reattendances and reduction in the average number of outpatient clinical attendances per referral. Finger et al [28] demonstrated no significant difference in upper extremity disability, return to work, or satisfaction in patients assessed and provided with conservative treatment by a hand surgeon, then given optional or scheduled follow-up, for simple upper extremity fractures. Brogan et al. [29] described excellent outcomes of fifth metatarsal fractures and supports the use of a VFC model to provide standardised, high-quality, and cost-effective care. Furthermore, Gilbert et al. [23] demonstrated how quickly and successfully virtual clinics could be set up in the face of a global pandemic. The Logan Hospital results concurred with the findings of the previous authors that this is a safe, efficient way to satisfactorily manage self-limiting injuries to improve overall clinical efficiency.

Vardy et al. [5] have demonstrated that instigation of a standardised pathway of care for predetermined minor injuries not requiring admission, from diagnosis through to management and subsequent follow-up, will facilitate more timely injury management in the ED. The Logan Hospital care pathway reduced the use of plaster cast immobilisation in favour of removable generic splinting devices. These are more time efficient and requires less specific skills to apply than plaster/fibreglass cast, further reducing time to discharge from the ED. Implementation of these pathways and splinting also reduces the number of patients returning to the ED for plaster care issues or for follow-up after failing to attend a planned OFC appointment. $[5,11]$ Following implementation of the VFC model at Logan Hospital there were $22 \%$ less plaster 
technician appointments within the OFC, presenting a significant cost saving in consumables as well as decreasing time pressures upon the plaster technicians.

Implementation of a VFC model has significant cost saving potential for healthcare providers and may allow redistribution of funds to other services. [30] Whilst we anticipate significant savings for the hospital with the implementation of the VFC, a formal cost analysis was not conducted. A full health econometric analysis would also consider the costs of medical staff (doctors, nurses, radiographers, allied health practitioners and plaster technicians), consumables (removable splints, POP, cast fiberglass), and the cost savings encountered by reducing patient time spent the in ED.

Jayaram et al. [31] demonstrated high rates of patient satisfaction ( $87 \%$ satisfied) with Mason 1 and 2 radial head fractures managed in a VFC model. Our paper demonstrates that patients diverted to APHC from VFC show clinically and statistically significant improvements in QuickDASH scores. Furthermore, levels of satisfaction from the patients were very high with $99 \%$ of consumers being satisfied with diversion from OFC to APHC, the knowledge of the therapist and the treatment received. Although Jayaram et al. [31] reported $87 \%$ satisfaction, this was only with radial head fractures, and assessed satisfaction with the leaflet provided. To our knowledge this is the first paper reporting consumer satisfaction with the change in model of care, reporting satisfaction with not seeing an Orthopaedic doctor and reporting outcomes across multiple upper limb conditions.

The obvious area for concern with a VFC is the potential to miss a serious injury. Whilst VFC clinics have demonstrated that management of predefined injuries is safe $[7,8,27,29$, 32-34], additional steps were introduced at Logan Hospital to ensure patients were not harmed or treatment compromised, as this was the first clinic of its kind to be introduced to the region. Three additional steps were introduced at Logan Hospital. First was introduction of a VFC Hotline for patients and GPs with queries to directly communicate with an orthopaedic registrar. The second step relates to staffing of the VFC, as the clinic is run by junior orthopaedic registrars. To address this, the VFC is performed alongside a senior orthopaedic consultant clinic each morning, so the junior staff have a readily available avenue for support from senior staff. The third step was to introduce allied health as a VFC discharge location to ensure follow up of all discharged patients. This was a modification to the VFC at Glasgow Royal Infirmary [9]; we discharge a large percentage of VFC patients to allied health as we feel this not only improves their rehab and recovery but is an additional step to prevent patients from "falling through the cracks" in the community should a serious injury be missed initially. This was necessitated due to the difference in primary care access in the United Kingdom compared to Australia. Patients are registered with a single GP in the UK thus enabling an easily identifiable follow up safety net. In Australia, patients may not be registered with a GP or may be registered at multiple sites, making primary care follow up less reliable.

\section{CONCLUSION}

Overall, the implementation of a VFC at our hospital was successful in improving efficiency and reducing the current OFC workload, as well as having a positive effect on the FTA rate. Diversion to $A P H C$ resulted in clinically and statistically significant improvements in outcomes measures, satisfied consumers, and reduction in clinic load and plaster technician workload allowed additional time to be spent with complex patients, prevented clinic backlogs and overbooking with associated crowding of waiting rooms. We have also demonstrated that the Glasgow Hospital VFC model can be successfully modified to suit different hospital regions and patient demographics.

We anticipate this service will continue to improve and will reduce the workload of both the OFC and the ED for the management of minor injuries. In an era dealing with a global pandemic that is spread via person-to-person direct contact, the virtues of a virtual clinic are exponential. This model of care has been adopted permanently in our hospital. We are optimistic that VFC models are a socially distanced, pandemic-proof clinical care model that will be the way of the future for Australian orthopaedic departments.

\section{DISCLOSURE STATEMENT}

The authors declare that they have no relevant or material financial interested that relate to the research described in this paper and have nothing to disclose. 


\section{Referebces}

1. Services DoHH. Specialist Clinics Service Improvement Guide. 2013.

2. Getting It Right First Time Queensland - Orthopaedic State Report. State of Queensland (Queensland Health); October 2020.

3. Cleary A, Zeller R, Maguire C, Goh S, Shortt N. Do all adult orthopaedic injuries seen in emergency departments need to attend fracture clinic? A Queensland multicentred review. Emerg Med Australas. 2017;29(6):658-63.

4. Waters S, Edmondston SJ, Yates PJ, Gucciardi DF. Identification of factors influencing patient satisfaction with orthopaedic outpatient clinic consultation: A qualitative study. Man Ther. 2016;25:48-55.

5. Vardy J, Jenkins PJ, Clark K, Chekroud M, Begbie K, Anthony l, et al. Effect of a redesigned fracture management pathway and 'virtual' fracture clinic on ED performance. BMJ Open. 2014;4(6):e005282.

6. Legg P, Ramoutar D, Shivji F, Choudry B, Milner S. The construction and implementation of a clinical decision-making algorithm reduces the cost of adult fracture clinic visits by up to pound 104,800 per year: a quality improvement study. Ann R Coll Surg Engl. 2017:99(4):280-5.

7. Little M, Huntley D, Morris J, Jozsa F, Hardman J, Anakwe RE. The virtual fracture clinic improves quality of care for patients with hand and wrist injuries: an assessment of 3709 patients. J Hand Surg Eur Vol. 2020;45(7):748-53.

8. O'Reilly M, Breathnach O, Conlon B, Kiernan C, Sheehan E. Trauma assessment clinic: Virtually a safe and smarter way of managing trauma care in Ireland. Injury. 2019;50(4):898-902.

9. Jenkins P, Gilmour A, Murray O, Anthony I, Nugent M, Ireland AJ, et al. The Glasgow Fracture Pathway: a virtual clinic. BJJ News. 2014;2:22-4.

10. Logishetty K. Adopting and sustaining a Virtual Fracture Clinic model in the District Hospital setting - a quality improvement approach. BMJ Qual Improv Rep. 2017;6(1).

11. Cavka B, Cross E, Montvida O, Plunkett G, Oppy A, Bucknill A, et al. Retrospective cohort study evaluating the efficacy and safety of an orthopaedic consultantled virtual fracture clinic in an Australian level 1 trauma centre. ANZ J Surg. 2021.
12. Truter P, Shales S, Evans R, Giglia-Smith L, O'Hanlon S, Yates $P$. Virtual Fracture Clinic pilot in an orthopaedic tertiary hospital setting: patient characteristics, clinical contact metrics and operational challenges. Tasman Medical Journal. 2021;3(1):30-6.

13. Tsang P, Walton D, Grewal R, MacDermid J. Validation of the QuickDASH and DASH in Patients With Distal Radius Fractures Through Agreement Analysis. Archives of Physical Medicine and Rehabilitation. 2017;98(6):1217-22.

14. Bernal JL, Cummins S, Gasparrini A. Interrupted time series regression for the evaluation of public health interventions: a tutorial. Int J Epidemiol. 2017;46(1):34855.

15. Wagner AK, Soumerai SB, Zhang F, Ross-Degnan D. Segmented regression analysis of interrupted time series studies in medication use research. J Clin Pharm Ther. 2002;27(4):299-309.

16. Team RC. R: A language and environment for statistical computing. Vienna, Austria: R Foundation for Statistical Computing; 2020.

17. Team R. RStudio: Integrated Development Environment for R. Boston, MA: RStudio, PBC; 2021.

18. The its.analysis R package - Modelling Short Time Series Data [Internet]. 2019. Available from: https://ssrn.com/abstract=3398189 or http://dx.doi.org/10.2139/ssrn.3398189.

19. Muggeo VMR. Interval estimation for the breakpoint in segmented regression: a smoothed score-based approach. Aust N Z J Stat. 2017;59(3):311-22.

20. Muggeo VMR. segmented: An R Package to Fit Regression Models with Broken- Line Relationships. $R$ News. 2008;8.

21. Koff E, Lyons N. Implementing value-based health care at scale: the NSW experience. The Medical Journal of Australia. 2020;212(3).

22. Speerin R, Needs C, Chua J, Woodhouse LJ, Nordin M, McGlasson R, et al. Implementing models of care for musculoskeletal conditions in health systems to support value-based care. Best Practice \& Research Clinical Rheumatology. 2020;24.

23. Gilbert AW, Billany JCT, Adam R, Martin L, Tobin R, Bagdai S, et al. Rapid implementation of virtual clinics due to COVID-19: report and early evaluation of a quality improvement initiative. BMJ Open Qual. 2020;9(2). 
24. Maheshwari K, Hindocha S, Yousif A. Virtual clinics: Need of the hour, a way forward in the future. Adapting practice during a healthcare crisis. J Plast Reconstr Aesthet Surg. 2020;73(7):1357-404.

25. McKirdy A, Imbuldeniya AM. The clinical and cost effectiveness of a virtual fracture clinic service: An interrupted time series analysis and before-and-after comparison. Bone Joint Res. 2017;6(5):259-69.

26. Murray O, Christen K, Marsh A, Bayer J. Fracture clinic redesign: improving standards in patient care and interprofessional education. Swiss Med Wkly. 2012;142:W13630.

27. Holgate J, Kirmani S, Anand B. Virtual fracture clinic delivers British Orthopaedic Association compliance. Ann R Coll Surg Engl. 2017;99(1):51-4.

28. Finger A, Teunis T, Hageman MG, Thornton ER, Neuhaus $V$, Ring D. Do patients prefer optional followup for simple upper extremity fractures: A pilot study. Injury. 2016;47(10):2276-82.

29. Brogan K, Bellringer S, Akehurst H, Gee C, Ibrahim N, Cassidy $L$, et al. Virtual fracture clinic management of fifth metatarsal, including Jones', fractures is safe and cost-effective. Injury. 2017;48(4):966-70.
30. Jenkins PJ, Morton A, Anderson G, Van Der Meer RB, Rymaszewski LA. Fracture clinic redesign reduces the cost of outpatient orthopaedic trauma care. Bone Joint Res. 2016:5(2):33-6.

31. Jayram P, Bahattacharyya, R., Jenkins, PJ., et al. Patient satisfaction following management of radial head and neck fractures in a virtual fracture clinic. J Shoulder Elbow Surg. 2014(23):297-301.

32. Bellringer SF, Brogan K, Cassidy L, Gibbs J. Standardised virtual fracture clinic management of radiographically stable Weber B ankle fractures is safe, cost effective and reproducible. Injury. 2017;48(7):1670-3.

33. Bhattacharyya $R$, Jayaram $P R$, Holliday $R$, Jenkins $P$, Anthony I, Rymaszewski L. The virtual fracture clinic: Reducing unnecessary review of clavicle fractures. Injury. 2017;48(3):720-3.

34. Robertson A, Godavitarne C, Bellringer S, Guryel E, Auld F, Cassidy L, et al. Standardised virtual fracture clinic management of Achilles tendon ruptures is safe and reproducible. Foot Ankle Surg. 2019;25(6):782-4. 